\title{
COMMUTATIVITY OF INTERTWINING OPERATORS. II
}

\author{
BY A. W. KNAPP1
}

Communicated by James Bramble, September 24, 1975

In a series of papers written partly in collaboration with E. M. Stein, the author has investigated the question of reducibility for the major unitary representations of a connected semisimple Lie group $G$ of matrices. These representations are induced representations of a particular kind from a cuspidal parabolic subgroup and are the ones that appear in the Plancherel formula for $G$ (see [1]). They split into at most finitely many irreducible pieces. One theorem in [7] is a dimension formula for the commuting algebra of such a representation. We can now give the full algebra structure of the commuting algebra, finding in particular that the irreducible constituents of each of the representations are inequivalent.

Let $M A N$ be the Langlands decomposition of a parabolic subgroup of $G$; the subgroup is cuspidal if $M$ has a discrete series. In this case let $\xi$ be a discrete series representation of $M$, let $\lambda$ be a unitary character of $A$, and let $U(\xi, \lambda, g)$ be the unitary representation of $G$ induced from the representation of $M A N$ given by man $\rightarrow \lambda(a) \xi(m)$. The collection of all the $U(\xi, \lambda, \cdot)$ is the set of representations that we consider. Let $C(\xi, \lambda)$ be the commuting algebra of $U(\xi, \lambda, \cdot)$.

THEOREM. Let $G$ be a connected semisimple Lie group of matrices, MAN a cuspidal parabolic subgroup, $\xi$ a discrete series of $M$, and $\lambda$ a unitary character of $A$. Then the commuting algebra $\mathrm{C}(\xi, \lambda)$ of $U(\xi, \lambda, \cdot)$ has a linear basis consisting of a set of unitary operators that form a group isomorphic to a direct sum of $r$ copies of $\mathbf{Z}_{2}$ where $r \leqslant \operatorname{dim} A$. In particular, $C(\xi, \lambda)$ is commutative.

This theorem was obtained earlier [3] , [4] for the case that $M A N$ is a minimal parabolic subgroup, i.e., one with $A$ as large as possible. The proof in the general case is considerably more complicated but does have points of contact with the proof in the minimal case. The main distinction is that $M$ is generated by its identity component and center in the minimal case but not in the general case.

The operators that form the linear basis in the theorem are of a particular form; namely they are the intertwining operators listed in Theorem 2(ii) of [7]. The number $r$ is easily computed, provided one knows some place where $\xi$ can be imbedded infinitesimally in the nonunitary principal series of $M$; this proviso

AMS (MOS) subject classifications (1970). Primary 22E30, 22 E45.

1 Supported by NSF grant MPS75-07024. 
is met if $M A N$ is a minimal parabolic or if, as often happens, the identity component of $M$ is the sum of a number of copies of $\operatorname{SL}(2, \mathbf{R})$.

The proof consists in reducing the theorem to the case that $G$ is split over $\mathbf{R}$ and $M A N$ is a minimal parabolic; this case was handled in [3] and [4]. In order to explain matters, it is necessary to recall some of the algebraic material in [7]. We shall omit in this discussion several pathological cases in which $\operatorname{dim} A$ $=1$ and one case in which $\operatorname{dim} A=2$; this last case must be investigated separately.

The Weyl group $W(A)$ is the normalizer of $A$ divided by the centralizer of $A$. By [5] it is the Weyl group of the root system $\Delta$ of useful roots of $A$. It acts on $\lambda$ and the class of $\xi$ by conjugation of the variable, and we let $W_{\xi, \lambda}$ be the subgroup that leaves $\lambda$ and the class of $\xi$ fixed. To each member of $W_{\xi, \lambda}$, [7] associates an intertwining operator that is a member of $C(\xi, \lambda)$. The group splits as a semidirect product $W_{\xi, \lambda}=W_{\xi, \lambda}^{\prime} R_{\xi, \lambda}$ with $W_{\xi, \lambda}^{\prime}$ normal, and the operators corresponding to $R_{\xi, \lambda}$ form the basis of $C(\xi, \lambda)$ that we work with. (The operators corresponding to $W_{\xi, \lambda}^{\prime}$ are all scalar.)

It is enough to prove that $R_{\xi, \lambda}=\Sigma Z_{2}$. This result does not fully prove the theorem, but the remaining details are handled as in [4], except for minor changes. In proving $R_{\xi, \lambda}=\Sigma Z_{2}$, it is enough to handle $\lambda=1$, showing $R_{\xi, 1}=$ $\Sigma \mathbf{Z}_{2}$.

The problem then is to understand the subgroup $W_{\xi, 1}$ of $W(A)$ that leaves the class of $\xi$ fixed. The restriction of $\xi$ to the identity component $M_{0}$ of $M$ is the sum of inequivalent discrete series, corresponding to an unordered set $\Lambda=$ $\left\{\mu_{i}\right\}$ of discrete series parameters. The class of $\xi$ is determined by $\Lambda$ and the restriction of $\xi$ to the center of $M$. The idea is to wash away all effects due to $\Lambda$, and then the remaining behavior of $\xi$ can be captured by referring matters to a minimal parabolic in a suitable group split over $\mathbf{R}$.

To accomplish this, let $W_{e}$ be the subgroup of $W(A)$ generated by the simple reflections $p_{\alpha}$ of $\Delta$ such that $\alpha$ has even multiplicity and $2 \alpha$ is not a root of $A$. Then $W(A)$ is a semidirect product $W(A)=W_{e} S$, where members of $S$ act as the identity on a compact Cartan subgroup of $M_{0}$. Clearly $S$ has no effect on $\Lambda$. The hardest step in the proof of the theorem is the following.

Lemma. The subgroup $W_{e, \Lambda}$ of $W_{e}$ that leaves the unordered set $\Lambda$ fixed is generated by reflections.

Now $W_{\Lambda}=W_{e, \Lambda} S$ is the full subgroup of $W(A)$ that leaves $\Lambda$ fixed, and it follows from the lemma, from Lemma 18(2) of [2], and from a little extra argument that $W_{\Lambda}$ is the Weyl group of a reduced root system $\Delta_{\Lambda}$. Let $G_{\Lambda}$ be a split group with $\Delta_{\Lambda}$ as root system and with complexification simply connected, and define 


$$
\sigma_{\Lambda}\left(\gamma_{\alpha}\right)= \begin{cases}+1 & \text { if } \mu_{\xi, \alpha}(0)=0 \\ -1 & \text { if } \mu_{\xi, \alpha}(0) \neq 0\end{cases}
$$

(See [6] for the definition and properties of $\mu_{\xi, \alpha}{ }^{2}$ and see [5] for the definition of $\gamma_{\alpha}$.) We can show that $\sigma_{\Lambda}$ is a well-defined character of the (finite abelian) group $M_{\Lambda}$ associated to a minimal parabolic subgroup of $G_{\Lambda}$. It follows readily that $R_{\xi, 1}$ is isomorphic with a subgroup of $R_{\sigma_{\Lambda}, 1}$, and the latter is $\Sigma \mathbf{Z}_{2}$ by [3].

\section{REFERENCES}

1. Harish-Chandra, Harmonic analysis on semisimple Lie groups, Bull. Amer. Math. Soc. 76 (1970), 529-551. MR 41 \#1933.

2. On the theory of the Eisenstein integral, Conference on Harmonic Analysis, Lecture Notes in Math., vol. 266, Springer-Verlag, Berlin and New York, 1972, pp. 123149.

3. A. W. Knapp, Determination of intertwining operators, Proc. Sympos. Pure Math., vol. 26, Amer. Math. Soc., Providence, R. I., 1973, pp. 263-268. MR 49 \#3032.

4. - Commutativity of intertwining operators, Bull. Amer. Math. Soc. 79 (1973), 1016-1018. MR 48 \#11399. $275-294$.

5. - Weyl group of a cuspidal parabolic, Ann. Sci. École Norm. Sup. 8 (1975),

6. A. W. Knapp and E. M. Stein, Singular integrals and the principal series. III, Proc. Nat. Acad. Sci. U. S. A. 71 (1974), 4622-4624.

7. - Singular integrals and the principal series. IV, Proc. Nat. Acad. Sci. U. S. A. 72 (1975), 2459-2461.

DEPARTMENT OF MATHEMATICS, CORNELL UNIVERSITY, ITHACA, NEW YORK 14853

2 If $\beta$ and $2 \beta$ are roots of $A, \Delta_{\Lambda}$ is to include $2 \beta$. We adopt the convention that $\mu_{\xi, 2 \beta}$ means the same thing as $\mu_{\xi, \beta}$. 